\title{
s. \\ Performance Analysis of Wavelet on Image Compression
}

\author{
${ }^{1}$ Pranoti Khollam, ${ }^{2}$ P. H. Mohite \\ ${ }^{1,2}$ Electronics and Telecommunication Department, SPPU, Pune University, Modern Education Society's College of \\ Engineering Pune 411001, India
}

\begin{abstract}
In this paper we viewed an approach for image compression using DWT technique. The DWT technique is explained with its algorithm, advantages. This technique has features like simplicity, handling complex kind of information, cost deduction in data storage, comparatively more fast than other compression algorithm, transmission on networks without major reduction in quality of the images.
\end{abstract}

Keywords - Wavelet, sub band, threshold

\section{INTRODUCTION}

Now image compression is not only need for efficient storage but also for its efficient transmission on the network. We are using this technique for still images. The images having format like PGM, PPM, BMP can be used to compress them.

The DWT dills with complex kind of information like is based on concepts like videos, music and various patterns into the elementary form.

The performance parameters like MSE, PSNR, SNR Compression Ratio tells us performance analysis of algorithm We are taking parameters MSE, PSNR , Compression Ratio for analysis.

\section{BLOCK DIAGRAM: DWT ENCODER}

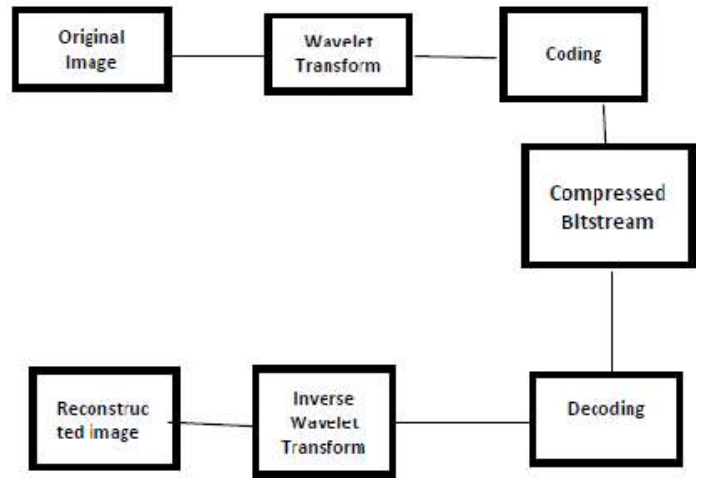

Fig.1. Block diagram of DWT

\section{1) Original Images}

We have taken Baboon512.pgm and Baboon512.ppm as original image for the compression of image. we need to take the original images in which intensity levels are uniformly distributed and high contrast.
2) Wavelet Transform

It splits the signal into a number of basis function. These basis function are called wavelets. Discrete wavelet transform convert the discrete time based signal into a discrete wavelet coefficients representation.

It is also based on Time and Scale representation. Applying Discrete wavelet transform (DWT) to 2-D image for compression. It can extract and gives information from various signals effectively and efficiently.

Multiresolution Analysis(MRA)

In this a complete image is splited into levels. The levels can be 1,2,3,4 and can be more.

Spatial distributions

After applying WT for first time to the various formats of images, the distributions (spatial) of wavelet coefficients have a better relationships with the original input images. There are four subband which describes our original single one image from various anglesa.s.Fig. 2

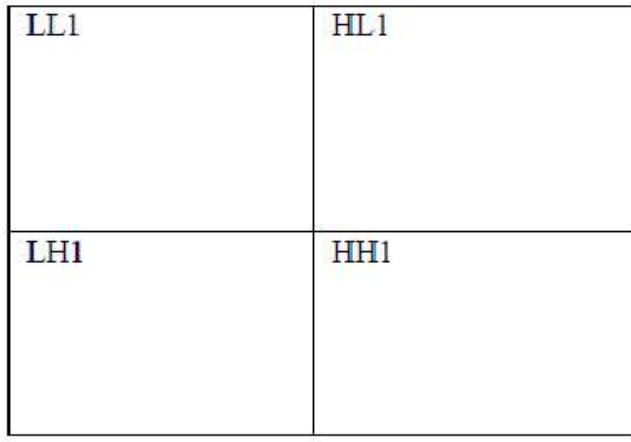

Fig.2.Frequency distribution after first WT 


\begin{tabular}{|l|l|l|}
\hline LL2 & HL2 & HL1 \\
\hline LH2 & HH2 & \\
\hline LH1 & HH1 \\
& & \\
\hline
\end{tabular}

Fig.3.Frequency distribution after second WT

But the more energy is stored in Lower Lower band while the specific details for the images can be found Higher Lower, Lower Higher, Higher Higher band. Further the high energy Lower Lower band can be splited a.s.Fig.3

\section{ALGORITHM}

1Digitization of input image into the signal which is matrix

2.Select wavelet levels

3.Decompsing the coefficients.4.Selecting signal into wavelets wavelets.

\section{Apply entropy coding}

\section{PERFORMANCE PARAMETERS}

1. PSNR: Peak signal to noise ratio

It is ratio of signal power to the noise power.

It should be maximum.

\section{CG: Compression gain}

It is uncompressed to the compressed bits achievement result.

It should me maximum.

\section{RESULTS}

Fig.4. Compression vs Percentage threshold

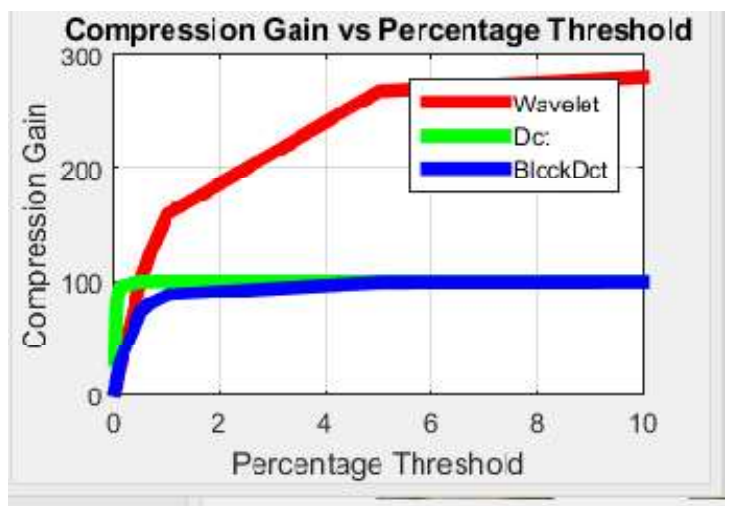

Fig.5.Compression gain vs Percentage threshold

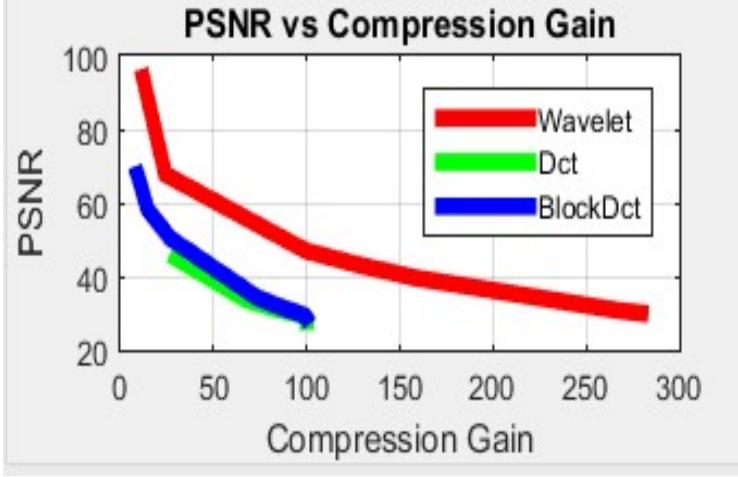

From fig.4. we concluded that for wavelet the compression gain is more than det and block det.

For various percentage of threshold, compression gain is increasing.

From fig.5. we concluded that for wavelet the PSNR is more than det and block det.

With increase in compression gain, PSNR gets reduced which is very essential.

\section{ADVANTAGES}

1. It has High Peak to signal noises ratios, Low mean squared errors

2. It is easy, efficient algorithm.

3. Reduces time \& cost in image storage

4. It reduces large number of bits in the transmission.

5. DWT more fast than other compression techniques.

6. Transmission on the network without major reduction in quality of the images.

\section{CONCLUSION}

From graphical representation of parameters, we concluded that dwt is most suitable for image compression

For the comparison of our compression technique with the other compression techniques, we have taken the DCT and Block DCT of the pgm, ppm extension image.

\section{REFERENCES}

[1] "Embedded Image Coding Using Zerotrees of Wavelet Coefficients", by Shapiro in the IEEE Transaction . on the Signal Processing, Volume. 41, pp 3445-3462, 1993.

[2] "Design of Image Compression Algorithm Using Matlab", by Abhishek Thakur, Rajesh Kumar, Amandeep Bath, Jitender Sharma in IJEEE, Vol. 1, Issue 1 (Jan-Feb 2014).

[3] "Visually Improved Image Compression by using Embedded Zero-tree Wavelet Coding ", by Janaki. $\mathrm{R}$ and Dr. Tamilarasi. A in IJCSI International Journal of the CS Issues, Volume. 8, Issue no2, March 11. 
[4] John G. Proakis, Dimitris G. Manolakis, "Digital Signal Processing: Principles, approach ", Pearson Publication.

[5] R. A. DeVore, B. Jawerth, and B. J. Lucier, "Image Compression Through Wavelet Transform Coding," IEEE Transaction. On Information Theory, Volume. 38, No. 2,pp. 719 746, 1992.
[6] S. Pemmaraju and S. Mitra, "Efficient image coding using multiresolution wavelet transform and vector quantization" IEEE Southwest topic on Image Analysis and Interpretation.

[7] Rafcel, Eddins,'DIP' by PE

[8] S.Jayremen,', DIP' by TMGHP 